Canad. Math. Bull. Vol. 21 (4), 1978

\title{
PROPERTIES OF HEREDITARY HYPERGRAPHS AND MIDDLE GRAPHS
}

\author{
BY
}

\author{
E. J. COCKAYNE, S. T. HEDETNIEMI, AND D. J. MILLER
}

\begin{abstract}
The middle graph of a graph $G=(V, E)$ is the graph $M(G)=\left(V \cup E, E^{\prime}\right)$, in which two vertices $u, v$ are adjacent if either $u$ is a vertex in $V$ and $v$ is an edge in $E$ containing $u$, or $u$ and $v$ are edges in $E$ having a vertex in common. Middle graphs have been characterized in terms of line graphs by Hamada and Yoshimura [7], who also investigated their traversability and connectivity properties. In this paper another characterization of middle graphs is presented, in which they are viewed as a class of intersection (representative) graphs of hereditary hypergraphs. Graph theoretic parameters associated with the concepts of vertex independence, dominance, and irredundance for middle graphs are discussed, and equalities relating the chromatic number of a graph to these parameters are obtained.
\end{abstract}

1. Introduction. The line graph of any graph $G=(V, E)$ is the graph $L(G)=\left(E, E^{\prime}\right)$, the vertices of which correspond 1-1 with the edges of $G$, and two vertices are adjacent in $L(G)$ if and only if the corresponding edges in $G$ have a vertex in common. Similarly, the middle graph $M(G)=\left(V \cup E, E^{\prime}\right)$ has for its vertex set the union of the vertices and edges of $G$, and having two vertices $u$ and $v$ adjacent if and only if either $u$ is a vertex of $G$ and $v$ an edge of $G$ containing $u$, or $u$ and $v$ are adjacent edges in $G$. One can obtain the middle graph of $G$ by superimposing $L(G)$ on the graph $S(G)$, which is the subdivision graph of $G$, obtained by "inserting" a new vertex on each edge of $G$, cf. Fig. 1. Notice that if $u$ and $v$ are adjacent in $G$ then they are not adjacent in $M(G)$.

2. Characterizations. Middle graphs arise naturally in the study of representative graphs of hereditary hypergraphs. Recall that a hypergraph $H=(V, E)$ consists of a set of vertices $V$ together with a set of edges $E$, each of which is an arbitrary subset of $V$. A hypergraph is hereditary if $e_{1} \in E$ and $e_{2} \subseteq e_{1}$ implies $e_{2} \in E$. The representative graph of a hypergraph $H=(V, E)$ is the graph $\Omega(H)=\left(E, E^{\prime}\right)$, the vertices of which correspond 1-1 with the edges of $H$, and two vertices are adjacent in $\Omega(H)$ if and only if the corresponding edges in $H$ have a non-empty intersection. Stated in other terms, the representative graph $\Omega(H)$ is the intersection graph of the family $E$ of subsets of $V$. Although every graph $G=(V, E)$ is a hypergraph, this hypergraph is not hereditary. A natural

Received by the editors January 25, 1977 and, in revised form, September 8, 1977 and January 17, 1978. 


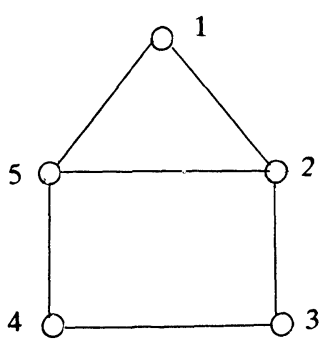

$G$

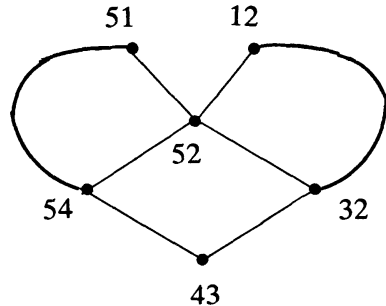

$L(G)$

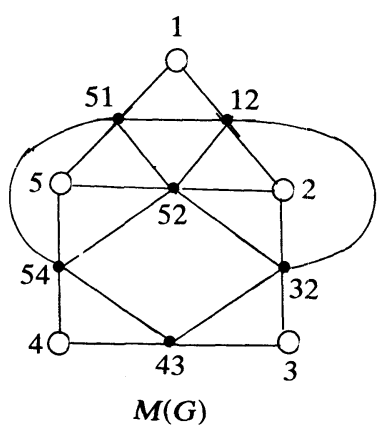

Figure 1.

hereditary hypergraph associated with $G$ is the hypergraph $H H(G)=$ $(V, V \cup E)$. It is easy to see that the representative graph of $H H(G)$, $\Omega(H H(G))$, is a middle graph, and conversely. Thus, for any graph $G, M(G) \simeq$ $\Omega(H H(G))$.

Hamada and Yoshimura [7], have shown that every middle graph is isomorphic to a line graph. If a graph $G^{+}$is constructed from a graph $G$ by adding to each vertex of $G$ an adjacent endvertex, then $M(G) \simeq L\left(G^{+}\right)$. This result can be used to obtain the following characterization of middle graphs. A clique $C$ of a graph $G$ is a maximal complete subgraph of $G$. A vertex of $G$ is called unicliqual if it is an element of only one clique; such a clique will be called a unicliqual clique. It is important to observe that a vertex $v$ is unicliqual if and only if the subgraph induced by the vertices adjacent to $v$ is complete. The following result due to Sampathkumar and Chikkodimath [12] characterize middle graphs.

Proposition 2.1. A graph $G$ is a middle graph if and only if the unicliqual cliques $C_{1}, C_{2}, \ldots, C_{k}$ of $G$ satisfy the following conditions:

(i) $\left|C_{i} \cap C_{j}\right| \leq 1$,

(ii) $C_{1}, C_{2}, \ldots, C_{k}$ is a partition of $E(G)$, and

(iii) every non-unicliqual vertex lies in exactly two cliques.

3. Isomorphisms. The following two remarks are almost immediate consequences of the fact that middle graphs form a subclass of the class of all line graphs.

REMARK 3.1. For any graphs $G_{1}$ and $G_{2}, G_{1} \simeq G_{2}$ if and only if $M\left(G_{1}\right) \simeq$ $M\left(G_{2}\right)$. To establish this remark we first note that $G_{1} \simeq G_{2}$ if and only if $G_{1}^{+} \simeq G_{2}^{+}$. By Whitney's Theorem on line graphs [15], $G_{1}^{+} \simeq G_{2}^{+}$if and only if $L\left(G_{1}^{+}\right) \simeq L\left(G_{2}^{+}\right)$, and since $L\left(G_{i}^{+}\right) \simeq M\left(G_{i}\right)$, the remark follows. It should be noted that the exceptional cases of Whitney's Theorem involving the graphs $K_{1,3}$ and $K_{3}$ do not occur here.

The automorphism group of a graph $G$ will be denoted by Aut $(G)$. 
REMARK 3.2. For any graph $G$, without isolated vertices, Aut $(G) \simeq$ Aut $(M(G))$. Since $G$ contains no isolated vertices it is easy to establish that Aut $(G) \simeq$ Aut $\left(G^{+}\right)$. Again we note that the exceptional cases of Whitney's Theorem involving the graphs $K_{1,3}$ and $K_{3}$ do not occur here and hence by Whitney's Theorem [15] and a theorem of Sabidussi [11, p. 365] we have that Aut $G \simeq$ Aut $\left(L\left(G^{+}\right)\right)$. But $L\left(G^{+}\right) \simeq M(G)$ and hence it follows that Aut $(G) \simeq$ Aut $(M(G))$.

4. Independence, dominance, and irredundance. Let $G$ be an undirected graph with vertex set $V$. If $v \in V$ the closed neighbourhood of $v$ consists of the set $N[v]=\{v\} \cup\{x \mid x$ is adjacent to $v\}$. The concepts of independence, dominance and irredundancy of vertex subsets of $G$ may be defined in terms of closed neighbourhoods.

A subset $I$ is independent if no vertex of $I$ lies in the closed neighbourhood of any other vertex of $I$; a subset $D$ is dominating if the union of the closed neighbourhoods of the vertices in $D$ is $V$, and a subset $R$ is irredundant if for each vertex $v$ of $R, N[v]$ is not contained in the union of all other closed neighbourhoods $N[u]$, with $u \in R, u \neq v$.

The properties of independence sets in graphs are well known. Dominating sets have been studied by Ore [10], Berge [1] and in [3, 4, 5]. Since the notion of irredundancy is possibly new, it is briefly discussed here.

In elementary terms, a subset $R$ of $V(G)$ is irredundant if the closed neighbourhood of each vertex of $R$ adds to $\bigcup_{r \in R} N[r]$ a vertex, which no other vertex of $R$ contributes. Figure 2 depicts a graph $G$; the table of which shows the closed neighbourhoods of the irredundant set $R=\{3,4,7\}$. The circled vertex in each neighbourhood $N[u]$, is a vertex which is in no other closed neighbourhood of vertices of $R$.

Note that any independent set is irredundant. A set is independent and dominating if and only if it is maximal independent [1, p. 309]. The following is an analogous result for irredundancy. The proof is obvious and is omitted.

Proposition 4.1. A set is irredundant and dominating if and only if it is minimal dominating.

We now define parameters $\operatorname{ir}(G)(\operatorname{IR}(G)), \gamma(G)(\Gamma(G)), i(G)\left(\beta_{0}(G)\right)$ to be the smallest (largest) orders of maximal irredundant, minimal dominating and

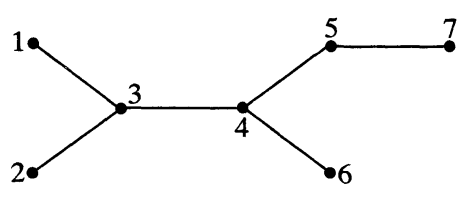

\begin{tabular}{l|cccc} 
& \multicolumn{4}{|c}{$N[u]$} \\
\hline 3 & 3 & 1 & 2 & 4 \\
4 & 4 & 3 & 5 & 6 \\
7 & 7 & 5 & &
\end{tabular}

Figure 2 . 
maximal independent sets of vertices of $G$, respectively. In general these parameters of a graph are distinct and by virtue of the above satisfy the following string of inequalities (proof omitted).

Proposition 4.2. For any graph $G$,

$$
\operatorname{ir}(G) \leq \gamma(G) \leq i(G) \leq \beta_{0}(G) \leq \Gamma(G) \leq I R(G) .
$$

The next results evaluate these parameters for representative graphs of hereditary hypergraphs.

5. Properties of hereditary hypergraphs. If $H=(X, E)$ is a hereditary hypergraph without isolated vertices (a vertex in no edge of $E$ ), define $\pi(H)$ to be the smallest order of a partition of $X$ into elements of $E$.

THEOREM 5.1. If $H$ is a hereditary hypergraph with $p$ vertices and no isolated vertices, then

$$
\operatorname{ir}(\Omega(H))=\gamma(\Omega(H))=i(\Omega(H))=\pi(H)
$$

and

$$
\operatorname{IR}(\Omega(H))=\Gamma(\Omega(H))=\beta_{0}(\Omega(H))=p .
$$

We now prove three propositions which will establish Theorem 5.1.

Proposition 5.2. If $H$ is a hereditary hypergraph with $p$ vertices and no isolated vertices, then $i(\Omega(H))=\pi(H)$ and $\beta_{0}(\Omega(H))=p$.

Proof. $X_{1}, \ldots, X_{k}$ is a partition of $X$ into edges of $H$ if and only if the corresponding set of vertices of $\Omega(H)$ is an independent dominating set of $\Omega(\mathrm{H})$.

Proposition 5.3. If $H$ is a hereditary hypergraph with $p$ vertices and no isolated vertices, then $\operatorname{IR}(\Omega(H))=p$.

Proof. Let $X_{1}, \ldots, X_{k}$ be irredundant vertices of $\Omega(H)$. Then for each $j \in\{2, \ldots, k\}, X_{j}-\bigcup_{i<j} X_{i} \neq \phi$ otherwise $N\left[X_{j}\right] \subseteq \bigcup_{i<j} N\left[X_{i}\right]$ contrary to the definition of irredundancy. Therefore $\left|\bigcup_{i \leq j} X_{i}\right| \geq j$ for each $j=2, \ldots, k$. Hence $p=\left|\bigcup_{i \leq k} X_{i}\right| \geq k$ and $\operatorname{IR}(\Omega(H)) \leq p$.

Conversely the properties of $H$ imply that $\{1\}, \ldots,\{p\}$ is an independent and hence irredundant set of vertices of $\Omega(H)$ and thus $\operatorname{IR}(\Omega(H)) \geq p$. This completes the proof of the proposition.

Proposition 5.4. If $H$ is a hereditary hypergraph without isolated vertices then $\operatorname{ir}(\Omega(H))=\pi(H)$. 
Proof. By $N[S]$ we shall mean the closed neighborhood in $\Omega(H)$ of the vertex $S$ of $\Omega(H)$. Let $X_{1}, \ldots, X_{k}$ be the edges of $H$ corresponding to the vertices of a maximal irredundant set of vertices of $\Omega(H)$. To establish that $\bigcup_{i=1}^{k} X_{i}=X$, assume the contrary, that is, there exists an $x \in X$ with $x \notin \bigcup_{i=1}^{k} X_{i}$. We show that $\left\{X_{1}, \ldots, X_{k},\{x\}\right\}$ is an irredundant set of vertices of $\Omega(H)$, contrary to maximality.

Firstly $N[\{x\}]$ contains $\{x\}$ which is not in $\bigcup_{i=1}^{k} N\left[X_{i}\right]$, since $x \notin X_{i}$. Suppose for some $j$,

$$
N\left[X_{j}\right] \subseteq \bigcup_{i \neq j} N\left[X_{i}\right] \cup N[\{x\}]
$$

Then the redundancy of $\left\{X_{1}, \ldots, X_{k}\right\}$ implies the existence of a vertex of $\Omega(H)$ i.e. an edge $W$ of $H$, such that

$$
W \in N\left[X_{j}\right], \quad W \in N[\{x\}] \text { and } W \notin \bigcup_{i \neq j} N\left[X_{i}\right]
$$

Therefore $x \in W, W \cap X_{j} \neq \phi$ and $W \cap X_{i}=\phi(i \neq j)$. Since $x \notin X_{j}$ we deduce that there exists $y \in W \cap X_{j}$ satisfying $y \notin X_{i}(i \neq j)$ and $y \neq x$. Hence

$$
\{y\} \in N\left[X_{j}\right]-\left(\bigcup_{i \neq j} N\left[X_{i}\right] \cup N[\{x\}]\right)
$$

contrary to (1). Therefore $\bigcup_{i=1}^{k} X_{i}=X$ as asserted. Define $Y_{1}=X_{1}$ and $Y_{j}=$ $X_{j}-\bigcup_{i<j} X_{i}(j=2, \ldots, k)$.

Then $Y_{j} \neq \phi$ for $j=1, \ldots, k$. Otherwise, some $X_{j} \subseteq \bigcup_{i<j} X_{i}$ which implies $N\left[X_{j}\right] \subseteq \bigcup_{i<j} N\left[X_{i}\right]$ contrary to irredundancy. By construction $Y_{1}, \ldots, Y_{k}$ is a partition of $X$ of order $k$ and the hereditary property implies that each $Y_{j}$ is an edge of $H$. Hence $\operatorname{ir}(\Omega(H)) \geq \pi(H)$.

Conversely if $X_{1}, \ldots, X_{k}$ is a partition of $X$ into edges of $H$, then the corresponding vertices of $\Omega(H)$ are independent and hence irredundant. Thus $\operatorname{ir}(\Omega(H)) \leq \pi(H)$, proving the proposition.

Theorem 5.1 follows immediately from Propositions 5.2, 5.3, and 5.4.

Let $X$ be the vertex set of a $p$ vertex graph $G$ and $E$ be the set of all independent sets of vertices of $G$. Then $H=(X, E)$ is a hereditary hypergraph whose representative graph is the independence graph $I(G)$ of $G$ studied in $[2,13]$ and $\pi(H)=\chi(G)$, the chromatic number of $G$. Theorem 5.1 specializes to the following corollary, part of which was established in [2].

COROLlary 5.5. For any graph $G$ having p vertices

$$
\begin{gathered}
\operatorname{ir}(I(G))=\gamma(I(G))=i(I(G))=\chi(G) \\
I R(I(G))=\Gamma(I(G))=\beta_{0}(I(G))=p .
\end{gathered}
$$


The theorem may be specialized to involve other graph theoretic parameters in precisely the same way as Theorem 6 of [2] was specialized.

Define $\alpha_{1}(G)$ as the minimum number of edges necessary to cover all the vertices of $G$.

THEOREM 5.6. For the hereditary hypergraph $H H(G)$ of any connected graph $G, \pi(H H(G))=\alpha_{1}(G)$.

Proof. Let $\pi=\left\{X_{1}, X_{2}, \ldots, X_{k}\right\}$ be a partition of minimum order of the vertices $V$ into edges of $H H(G)$. Let $X_{1}, X_{2}, \ldots, X_{i}$ be edges of $G$ and $X_{i+1}, \ldots, X_{k}$ be vertices of $G$. For each such vertex $X_{i}$, let $Y_{i_{1}}$ be any edge of $G$ incident with $X_{i,}$. Then $\left\{X_{1}, X_{2}, \ldots, X_{i}, Y_{i+1}, \ldots, Y_{k}\right\}$ is a set of edges of $G$ which covers all the vertices of $G$. Therefore,

$$
k=\pi(H H(G)) \geq \alpha_{1}(G) .
$$

Conversely, let $\left\{Y_{1}, Y_{2}, \ldots, Y_{k}\right\}$ be a minimum set of edges which contain all the vertices of $G$. Define $X_{j}=Y_{j}-\bigcup_{i<j} X_{i}$. Note that no $X_{j}=\phi$, since this would contradict the minimality of $\left\{Y_{1}, Y_{2}, \ldots, Y_{k}\right\}$. Therefore, $\pi=$ $\left\{X_{1}, X_{2}, \ldots, X_{k}\right\}$ is a partition of the vertices of $G$ into edges of $H H(G)$, and $\pi(H H(G)) \leq k=\alpha_{1}(G)$, completing the proof.

In the next section we shall use these results on hereditary hypergraphs to obtain several parameter values of middle graphs.

6. Properties of middle graphs. Theorems 5.1 and 5.6 and the observation that $\Omega(H H(G)) \simeq M(G)$ enable us to determine several parameter values of middle graphs.

THEOREM 6.1. For any connected graph $G$ with $p$ vertices and $q$ edges,

(i) $\operatorname{ir}(M(G))=\gamma(M(G))=i(M(G))=\alpha_{1}(G)$.

(ii) $\beta_{0}(M(G))=\Gamma(M(G))=I R(M(G))=p$, and

(iii) $\alpha_{0}(M(G))=q$.

It can be observed from Theorem 6.1 that for any connected graph $G=$ $(V, E)$ the set of vertices $V$ forms a maximum independent set of vertices, i.e. a $\beta_{0}$-set, in $M(G)$, and similarly the set $E$ forms a minimum edge cover, i.e. an $\alpha_{0}$-set, of $M(G)$. Equality (iii) above follows from (ii) and Gallai's result [6] that for any graph $G$ with $p$ vertices, $\alpha_{0}(G)+\beta_{0}(G)=p$, and the observation that $M(G)$ has $p+q$ vertices.

The precise determination of $\beta_{1}(M(G))$, the maximum number of independent edges in $M(G)$, can be made when $G$ is a tree or a unicyclic graph.

THEOREM 6.2. If $G$ is a connected graph of order $p$ with $q$ edges and at most one cycle, then $\beta_{1}(M(G))=q=\alpha_{0}(M(G))$. 
Proof. Since the maximum number of independent edges of a graph is at most half the number of vertices, then $\beta_{1}(M(G)) \leq(p+q) / 2<q$ when $p<q$ and $\beta_{1}(M(G)) \leq q$ when $G$ is a tree or a unicyclic graph, for which $p=q+1$ or $p=q$, respectively. In these two cases, one readily shows by induction on $q$ that $\beta_{1}(S(G))=q$, where $S(G)$ is the subdivision graph of $G$. Since $S(G) \subset M(G)$, then $\beta_{1}(S(G)) \leq \beta_{1}(M(G)) \leq q$, and the theorem follows, with the aid of Theorem 6.1(iii).

In [8], König showed that $\alpha_{0}(G)=\beta_{1}(G)$ holds when $G$ is bipartite. The proof of Theorem 6.2 shows that the same equality holds for middle graphs of forests and unicyclic graphs, but for no other middle graphs.

Our last parame iric result concerns the chromatic number $\chi$ and the size $\omega$ of a largest clique in a middle graph.

THEOREM 6.3. For any graph $G$ having maximum degree $d, \chi(M(G))=$ $\omega(M(G))=d+1$.

Proof. According to the theorem of Vizing [14] the chromatic number of the line graph $L(G)$ of a graph $G$ satisfies $d \leq \chi(L(G)) \leq d+1$.

Colour, therefore, the vertices of $M(G)$ which correspond to edges of $G$ with $d+1$ colours. Consider next colouring the vertices of $M(G)$ which correspond to vertices of $G$. Since each of these is adjacent in $M(G)$ to at most $d$ vertices, each may be assigned one of the $d+1$ colours. Thus, $\chi(M(G)) \leq d+1$. In Section 2 we noted that $M(G) \simeq L\left(G^{+}\right)$, and since $G^{+}$has maximum degree $d+1$, then $\omega\left(L\left(G^{+}\right)\right)=d+1$, so $d+1=\omega(M(G)) \leq \chi(M(G))$.

ACKNOWLEDGEMENTS. The authors would like to thank the referee for his numerous useful suggestions.

\section{REFERENCES}

1. C. Berge, Graphs and Hypergraphs, American Elsevier Inc., New York, 1973.

2. E. J. Cockayne and S. T. Hedetniemi, Independence Graphs, Proc. 5th S.E. Conf. on Combinatorics, Graph Theory and Computing (1974), 471-491.

3. E. J. Cockayne and S. T. Hedetniemi, Optimal Domination in Graphs, IEEE Trans. on Circuits and Systems Vol. CAS-22 No. 11 (1975), 855-857.

4. E. J. Cockayne and S. T. Hedetniemi, Towards a Theory of Domination in Graphs, Networks 7 (1977), 247-261.

5. E. J. Cockayne and S. T. Hedetniemi, Disjoint Independent Dominating Sets in Graphs, Discrete Math. 15 (1976), 213-222.

6. T. Gallai, Über extreme Punkt-und Kantenmengen, Ann. Univ. Sci. Budapest, Eotvos Sect. Math. 2 (1959), 133-138.

7. T. Hamada and I. Yoshimura, Traversability and Connectivity of the Middle Graph of a Graph, Discrete Math. 14 (1976), 247-255.

8. D. König, Graphen und Matrizen, Mat. Fiz. Lapok 38 (1931), 116-119.

9. J. Krausz, Démonstration nouvelle d'un théorème de Whitney sur les réseaux, Mat. Fiz. Lapok 50 (1943), 75-89.

10. O. Ore, Theory of Graphs, Amer. Math. Soc. Colloq. Publ. 38, Providence, 1962.

11. G. Sabidussi, Graph Derivatives, Math. Z. 76 (1961), 385-401. 
12. E. Sampathkumar and S. B. Chikkodimath, Semi-Total Graphs of a Graph-I, Karnatak Univ. Journal: Science-Vol. XVIII, 1973.

13. P. J. Slater, Irreducible Point Independence Numbers and Independence Graphs, Proc. 5th S.E. Conf. on Combinatorics, Graph Theory and Computing (1974), 647-660.

14. V. G. Vizing, On an Estimate of the Chromatic Class of a p-graph, Diskret. Analiz. 3 (1964), 25-30.

15 H. Whitney, Congruent Graphs and the Connectivity of Graphs, Amer. J. Math. 54 (1932), $150-168$.

DEPARTMENT OF MATHEMATICS

UNIVERSITY OF VICTORIA

VICTORIA, B.C. V8W 2Y2 